\title{
Minimally Invasive Oral Surgery Induction of the FRICT-ION Chronic Neuropathic Pain Model
}

\author{
Marena A. Montera ${ }^{\#}$ and Karin N. Westlund ${ }^{*}, \#$
}

Department of Anesthesiology \& Critical Care Medicine, University of New Mexico Health Sciences Center, Albuquerque, NM 87106, USA

*For correspondence: khigh@salud.unm.edu

\#Contributed equally to this work

\begin{abstract}
[Abstract] An easily induced preclinical trigeminal neuropathic nerve injury model is described here for the study of chronic pain, the model acronym FRICT-ION (Foramen Rotundum Inflammatory Constriction Trigeminal InfraOrbital Nerve). In patients, neuropathic pain is thought to be related to vascular alignment or multiple sclerosis along this small trigeminal nerve branch (V2) innervating the maxillary teeth and middle third of the face. With no detectable outward physical signs, the FRICT-ION model is ideal for blinded studies. The acronym FRICT-ION applied relates to the persistence of the trigeminal neuropathic pain model likely due to sliding irritation with normal chewing in the mice. A stepby-step method to induce the mild chronic rodent neuropathic pain model is described here. The surgery is performed orally through a tiny surgical slit inside the cheek crease to align a chromic gut suture irritant along the nerve as it passes into the skull. The model allows testing of non-evoked subjective measures and evoked quantitative mechanical hypersensitivity (allodynia) testing with von Frey filaments through at least 10-14 weeks (100 days). Anxiety and depression behaviors develop within 36 weeks relevant to the affective component of chronic pain. While many pain drugs have failed based on testing performed in the acute animal models available, the more stable and easily replicated trigeminal inflammatory compression model is the better suited for understanding both mechanistic and affective components of nerve injury-induced chronic neuropathic pain states as well as the more ideal for preclinical trials of novel non-opioid pain relief therapies.
\end{abstract}

Keywords: Chronic pain, Orofacial pain, Neuropathic pain, Neuralgia, Chromic gut, Infraorbital nerve, Anxiety

[Background] Damaged peripheral nerves cause persisting overactivation referred to as "neuropathic pain". A serious consequence of persisting nerve injury pain is the transition to chronic pain. Neuropathic pain is the result of changes in the signaling molecules in the peripheral nerve cells that eventually cause central sensitization and changes in the brain's pain and affective circuitry. A major obstacle to better understanding of pathophysiological mechanisms of chronic neuropathic pain and development of effective therapeutics is lack of available experimental animal models that mimic established chronic pain. To emulate chronic orofacial neuropathic pain, the FRICT-ION (Foramen Rotundum Inflammatory Constriction of Trigeminal InfraOrbital Nerve) nerve injury model was developed in mice to study behavioral, pharmacological, cellular, and molecular mechanisms persisting during chronic neuropathic pain. 
While many nerve injury models have been developed that allow study of nociceptive mechanisms and pain-related behaviors in the short term, the resilience of natural healing processes typically reverses nerve injury models within 3-4 weeks in neuropathic pain models. The method described below is one of the few models that allow long-term studies suitable for better understanding of clinical pain states and for testing experimental therapies. Several innate differences in the trigeminal nerve are likely responsible for the chronic persistence of the neuropathic model. The great importance of the trigeminal nerve to survival is postulated to be one characteristic responsible for the ease in producing persistence of trigeminal nerve injury pain and its effects on psychological well-being (Carlson, 2007). Anatomical proximity to the brain and the unique circuitry of the trigeminal system with one less synapse forming more direct connectivity with the limbic circuitry are also likely responsible for the intensity and persistence of neuropathic pain associated with the trigeminal nerve (Rodriguez et al., 2017).

Previous successful chronic trigeminal pain models described have been induced by loose chromic gut suture tie of the infraorbital nerve (ION) in rats (CCI-ION) behind the eye (Vos et al., 1994; Kniffin et al., 2015) or on the snout in rats (dloN-CCl) and mice (DIONI) (Ding et al., 2017; Hardt et al., 2019) (Figure 1). Alternatively, the intraoral approach has also been used to induce the $\mathrm{CCI}-\mathrm{ION}$ model in rats (Immamura et al., 1997). We previously introduced a trigeminal nerve hypersensitivity model induced by sliding the irritative chromic gut suture into the ION fissure behind the eye in mice creating a trigeminal inflammatory compression (TIC) (Figure 1) (Ma et al., 2012 and 2015; Lyons et al., 2015 and 2018). The surgical approach to the ION behind the eye is technically challenging and the vasculature prone to bleeding, often severely. After induction of trigeminal nerve injury models, mechanical hypersensitivity develops within a week and persists indefinitely in the ION's whisker pad receptive field. After trigeminal nerve injury, cognitive deficits are reported within 3 weeks in rats and anxiety- and depression-related symptoms emerge in subsequent weeks after injury ( $>6$ weeks) in rodents indicating brain origin and potential circuitry neuroplasticity within brain regions are responsible for these behaviors accompanying chronic pain (Yalcin et al., 2011 and 2014; Kniffin et al., 2015; Lyons et al., 2015 and 2018). Estimates are that in weeks 6-8 post-surgery, mice have experienced pain equivalent to 6-8 human years and persistence of models in this time frame can easily be considered chronic (Dutta and Sengupta, 2016; Hannaman et al., 2016). The interplay between psychologic and physical functioning is a particular consideration in patients with orofacial pain (Carlson, 2007). 


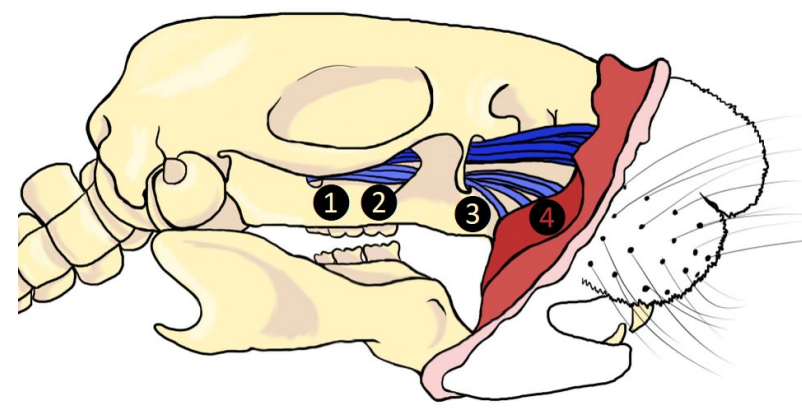

Figure 1. Surgical sites utilized in trigeminal neuropathic pain models. 1. TIC, 2. CCI-ION, 3. FRICT-ION, 4. dloN-CCI and DIONI.

Better models for the study of chronic neuropathic pain are needed to discover more effective treatments since the current treatment of choice, microvascular decompression surgery, in patients with chronic trigeminal nerve pain or multiple sclerosis pain wanes over time with reoccurrence typically at 3 years (Xia et al., 2014). The pain-free rate at 5 and 10 years decreases to $61 \%$ and $44 \%$ after decompression surgery to place a Teflon spacer and after stereotaxic radioablation is only $47 \%$, and $27 \%$, respectively (Wang et al., 2018). Teflon granulomas are reported in $5.6 \%$ of patients after microvascular decompression (Chen et al., 2000).

The FRICT-ION method provides a simpler intraoral approach (Figure 1). The benefits of using the mouse FRICT-ION model of trigeminal nerve injury for study of chronic neuropathic pain in general are many. The model induces long-term chronic neuropathic pain with no other apparent side effects or outward physical indication. The mouse experiences no health or weight gain issues. The method exposes the rodent to only minutes of isoflurane anesthesia but sensitivity in the receptive field on the whiskerpad persists indefinitely. If done correctly, the minimally invasive surgery has little or no bleeding. The model is quickly and easily mastered following the instructions provided below.

\section{Surgical Induction of the FRICT-ION Model}

We induce the model in either BALBc or C57BI/6 mice (20 to $25 \mathrm{~g}$; 8-10 weeks; Harlan Laboratories, Indianapolis, IN). We have performed the procedure in older mice with no complications. The surgery is performed open mouth through a tiny slit to align chromic gut suture along the trigeminal nerve. The constant but minimal nerve irritation likely produced with chewing is similar to one known cause of trigeminal neuropathic pain, irritation by proximity of the nerve to pulsating brainstem vasculature which is relieved by insertion of a Teflon sheet (Dandy, 1934; Xia et al., 2014). The extended duration of orofacial neuropathic pain models provides an optimal platform for preclinical testing of potential therapeutics since mechanical and cold sensitivities, anxiety, and depression-like behaviors are measurable.

The model illustrated here is induced by inserting $3 \mathrm{~mm}$ of chromic gut suture intraorally into the tight space where the infraorbital nerve (ION) passes through the bony infraorbital foramen. Similar to nerve 
biopsies from patients (Weis et al., 2012), the TIC model we reported previously using the intraorbital surgical approach did not cause the axonal degeneration seen in tied or cut nerve models (Ma et al., 2012). As a result, the TIC model induced hypersensitivities similar to those in the human condition, i.e., mechanical and cold allodynia, but not heat hypersensitivity, along with anxiety and depression. Though not tested as yet, we are hopeful that these results will be duplicated using the intraoral approach to induce the FRICT-ION model. The model is illustrated here induced on the right side of the mice in all figures. The model has been induced in both males and females, BALBc and C57BI/6, and while not yet tested by our lab, likely could also be done in rats. The intraoral approach has been used previously in rats to induce the nerve tied CCI-ION model (Immamura et al., 1997).

\section{Materials and Reagents}

1. $0.2 \mathrm{~cm}$ diameter corroborated evac tubing (Patterson Veterinary, catalog number: 07-8914311)

2. $1 \mathrm{~cm}$ diameter Tygon tube (Fisher Scientific, catalog number: 14-171-104)

3. Scavenger filter of the anesthesia unit (Patterson Scientific WAG unit, catalog number: 78909457)

4. Stabilization/tension ties constructed from rubber bands and surgical silk (shopmedvet.com, 50 silk; 100M cassette SKU: SLK50)

5. Embroidery floss (DMC, catalog number: 117S-3380)

6. Binder clip, ACCO 2" (Staples, catalog number: 72100)

7. Cotton-tipped applicators (shopmedvet.com, 6", non-sterile, SKU: CTA6)

8. Gloves

9. BALBc or C57BI/6 mice (20 to $25 \mathrm{~g}$; 8-10 weeks; Harlan Laboratories, Indianapolis, IN)

10. $70 \%$ ethanol (local source)

11. Sucrose

\section{Equipment}

1. 4-0 chromic gut suture (cut into $3 \mathrm{~mm}$ lengths) (Ethicon, catalog number: $635 \mathrm{H}$ )

2. Forceps (Dumont No. 5, Fine Science Tools, catalog number: 11251-10; Graefe Angled Serrated, Fine Science Tools, catalog number: 11049-10)

3. No. 11 scalpel blades (Feather/Electron Microscopy \#11, catalog number: 72044-11, VWR, catalog number:102097-822)

4. Scalpel handle \#3 (Walter Stern 320-051) (VWR, catalog number: 25607-947)

5. Bead sterilizer (Germinator 500 Glass Bead Sterilizer, Cell Point Scientific 5-1450) (VWR, catalog number: 101326-488)

6. Heating pad/recovery station for mice (HTP-1500 Adroit Veterinary Heat Pump and Soft Temp Veterinary Heat Pad V016) (Patterson Veterinary Supply, catalog numbers: 78910655 and 78910866) 
7. Surgery setup (see Procedure section below)

8. Dissecting scope (Optika SZN-10 Trinocular Stereo Zoom Microscope on Hinged Overhanging Stand, New York Microscope Company, catalog number: OPSZN-10)

9. Isoflurane anesthesia machine [Tec 3 EX Isoflurane Standard Fill Vaporizer, Versa II Anesthesia system, with Mapleson-D Non-Rebreathing (NRB) System] (Patterson Veterinary Supply, catalog numbers: 78703592, 78910545 with 78909636)

10. Base Plate, S\&T Ball Chain Retraction (Fine Science Tools, catalog number: 18100-00)

11. Fiber Optic Light (Dolan-Jenner Fiber-lite Illumination System 181-1) (VWR, catalog number: 41446-062)

12. Von Frey Filaments (Semmes-Weinstein, 20 monofilaments, BioSeb, bioseb.com)

13. Autoclave

\section{Software}

1. Ethovision XT8 videotracking system software (Noldus Information Technology, Leesburg, VA, USA) for analysis of movement in the affective behavioral tests

2. GraphPad Prism 8 for statistical analysis and data presentation (GraphPad Software Inc., La Jolla, CA)

\section{Procedure}

1. The surgery is performed on an alcohol cleaned flat surface beneath a dissection scope with an apparatus mounted with stabilization restraints to hold the mouth open. Recommendation is to use an S\&T Ball Chain Retraction System Base Plate. Two lengths of 5-0 surgical silk (or embroidery thread, soft string, to avoid damage to the mouse's tongue) with rubber bands knotted at the strings' ends can be hooked to the plate's ribbed edges. The surgical silk is drawn tight across the mouse's mouth for restraint of the mouth in an open position for anesthesia and surgery (Figure 2). Place the heating pad recovery station on a table adjacent to the surgery setup so that mice can be quickly transferred to the station upon completion of surgery. The station should be set to 41 degrees Celsius. We place an empty housing cage on top of the heating pad about an hour prior to the surgeries to allow the cage to heat up to a comfortable temperature; half on the pad, half off. 


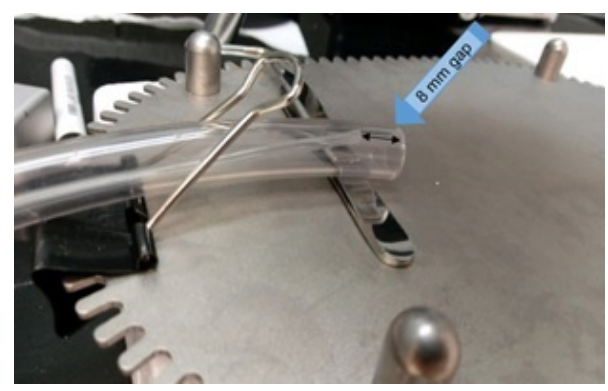

Figure 2. Mouse anesthesia setup

2. Since this is an oral surgery, a setup is needed that will reliably deliver isoflurane to the mouse while still leaving the mouth free for the operation. Recommendation is to use a two-tube system: a $0.2 \mathrm{~cm}$ diameter tube to deliver the anesthesia, slid inside a larger $1 \mathrm{~cm}$ diameter Tygon tube. The outer tube will connect to the scavenger filter of the anesthesia unit (Figure 2). Figure 3 provides further clarification of the orientation to accommodate the mouse's nose. There should be an $8 \mathrm{~mm}$ gap between the tip of the inner tube and the tip of the outer tube. The mouse's nose can be slid into the outer tube to allow anesthesia delivery, while still allowing the mouth to be opened and manipulated during surgery. The procedure is done under a dissection scope at a magnification of $10 x$, though higher magnifications might be beneficial when first learning the procedure.

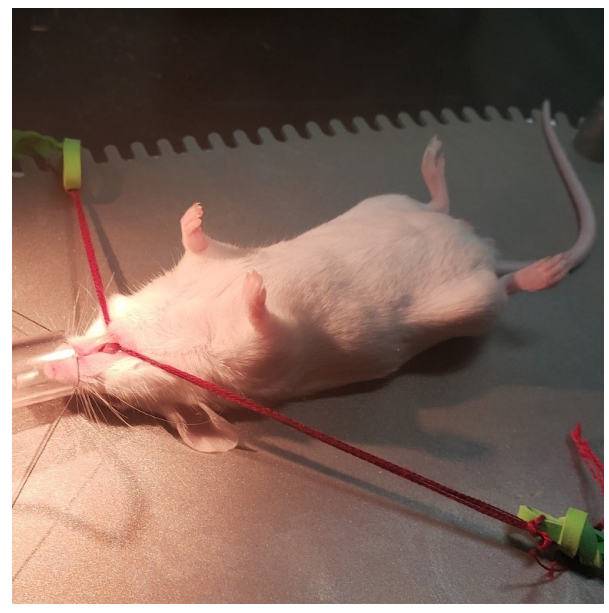

Figure 3. Mouse restraint

3. Sterilize surgery tools (scalpel handle, forceps) in a bead sterilizer, autoclave, or spray them with $70 \%$ ethanol and air dry.

4. Set up the anesthesia machine with a splitter to provide dual tubing approach of the mixed oxygen/isoflurane to both the induction chamber and the surgical setup. Optimal settings will depend on the isoflurane delivery vaporizer cannister, but current recommendation is an oxygen level of 1.5 and an isoflurane level of $3 \%$. 
5. Place the mouse into the induction chamber for initial anesthesia induction; the typical rapid breathing will begin to slow down to deeper breaths, moving from the upper chest to the lower chest. This will take about one to two minutes, depending on the mouse and the chamber size.

6. Switch anesthetic flow to the surgical area tubing.

7. Move the anesthetized mouse quickly to the surgical area beneath the dissection scope placing its nose into the Tygon tubing to maintain anesthesia depth and to avoid having to replace the mouse back into the induction chamber to repeat the anesthesia process. Should any movement occur, place the mouse back into the induction chamber and repeat this process. Movement of any kind by the mouse indicates the tubing is not providing adequate isoflurane flow.

8. Restrain the mouse for surgery. Hook one restraint under the upper two front teeth, and the other restraint underneath the lower two front teeth (Figure 4), gently pulling the mouth as wide open as possible while still keeping the mouse's nose inside the anesthesia tubing. If necessary, use tape across the tail to help hold the mouse steady.

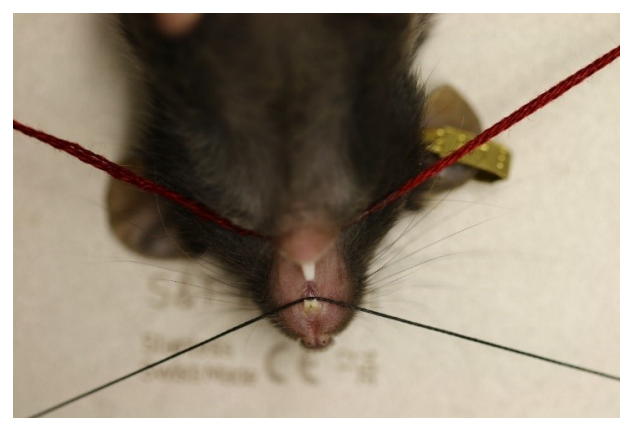

Figure 4. Top-down view of open-mouth positioning

9. Adjust the microscope and lighting as needed in order to have a clear visual into the mouse's mouth (Figure 5). From here, begin the procedure.

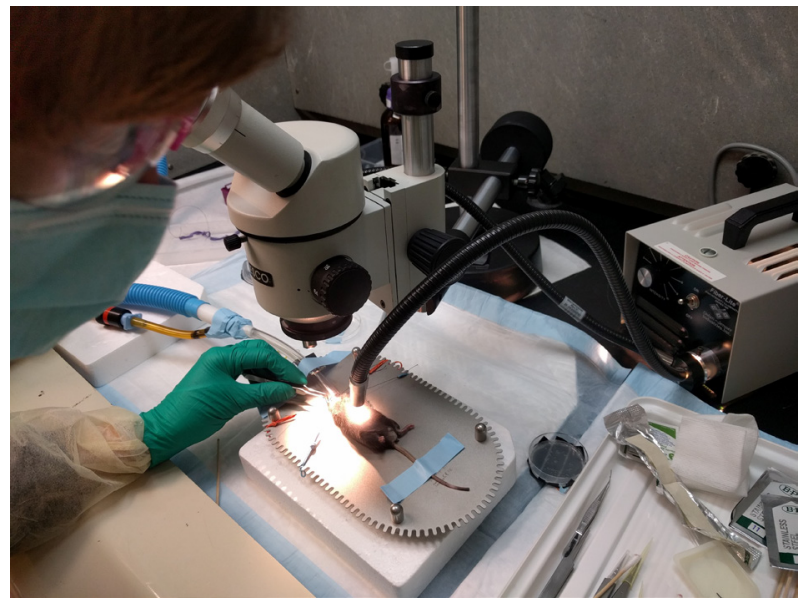

Figure 5. Broad view of the surgery and restraint set-up 
10. Using a scalpel blade (\#11) mounted on a surgical scalpel handle (\#3), make a small incision into the mouse's lip inner bucchal margin crease a little below the roof of the mouth (Figure 6).
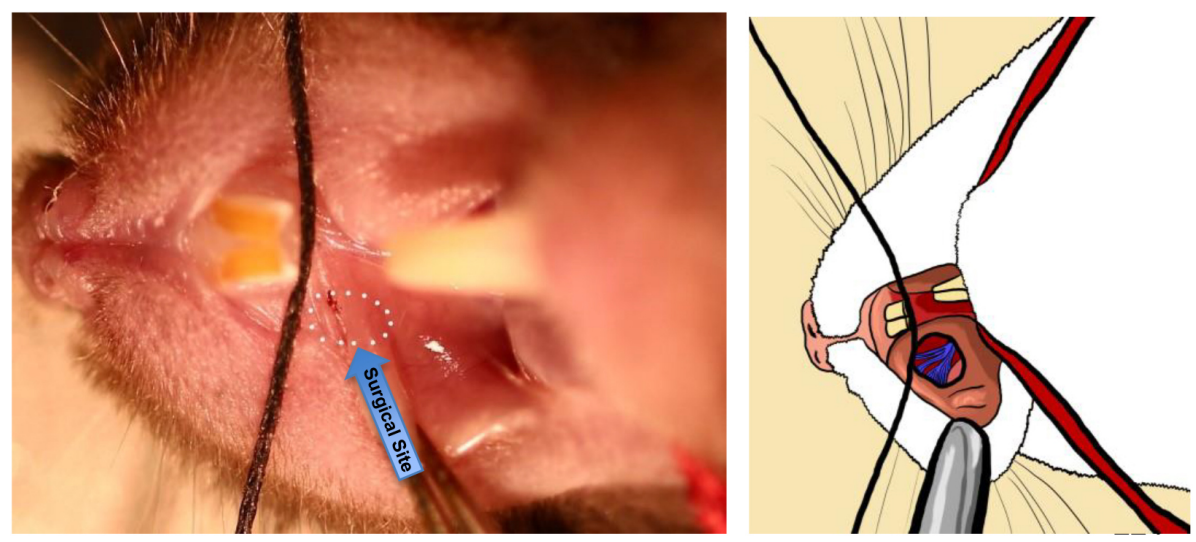

Figure 6. Highlighted surgical site provides illustration of the $2 \mathrm{~mm}$ incision (15x). The diagram reveals the underlying trigeminal nerve branches.

11. Gently cut away the soft tissue using the tip of the scalpel blade. You will eventually create a hole to reveal the trigeminal maxillary infraorbital nerve branches that innervate the teeth and whiskerpad coming together posteriorly at the bony foramen rotundum to enter the skull (Figure 7). Initially opening this site a little wider in a cadaver or practice mouse will allow visualization of the infraorbital nerve innervation and the foramen rotundum where the chromic gut will be inserted (Figures 8 and 9). The surgical site ordinarily should be quite small; no more than a couple of millimeters, and no suturing of the wound should be needed if an experienced experimenter did the surgery. Please note that the dissection and surgery have been exaggerated in all of the descriptive images provided. It is unlikely, but there would be excessive bleeding from the wound if the cut is deeper than intended, then stop the procedure and do not use the mouse for further experimentation.

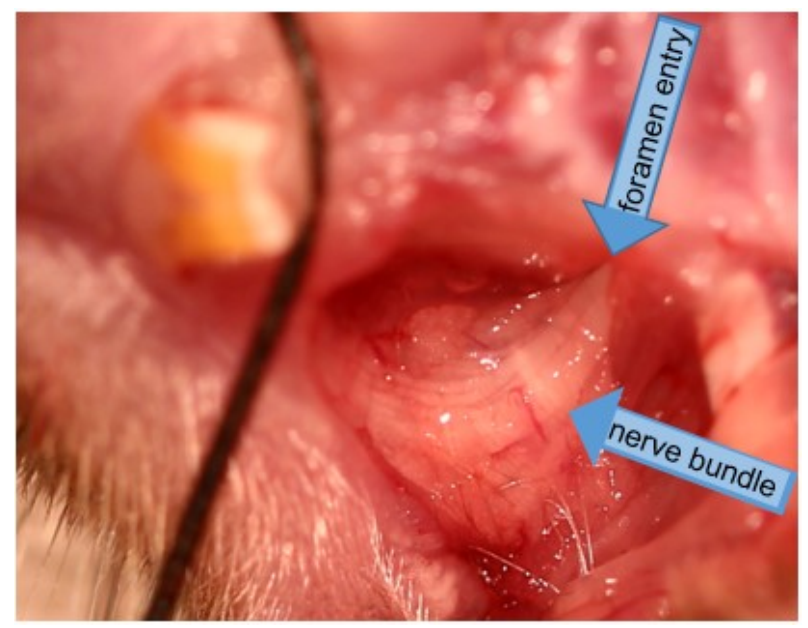

Figure 7. Demonstration of nerve bundle entering into foramen. The cut is exaggerated for better display in this higher power view (15x). 


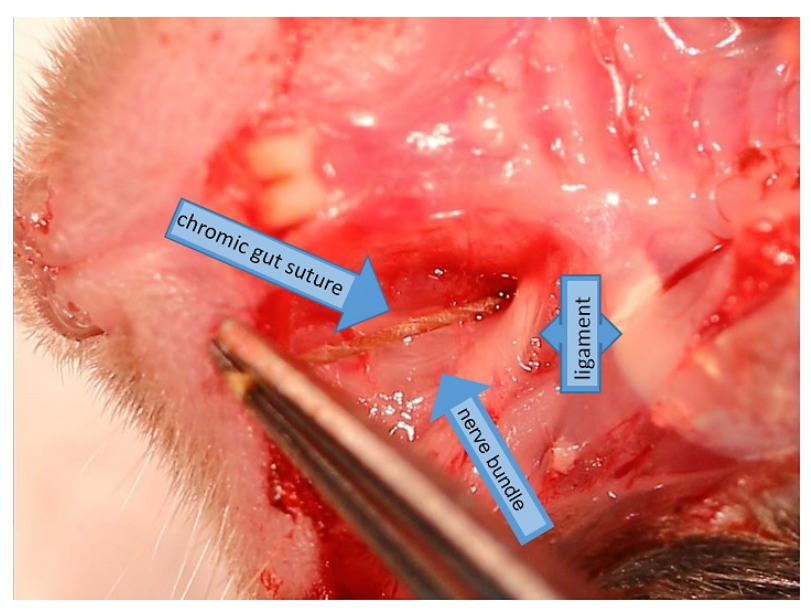

Figure 8. Demonstration of chromic gut suture placement within foramen exaggerated dissection (15x)

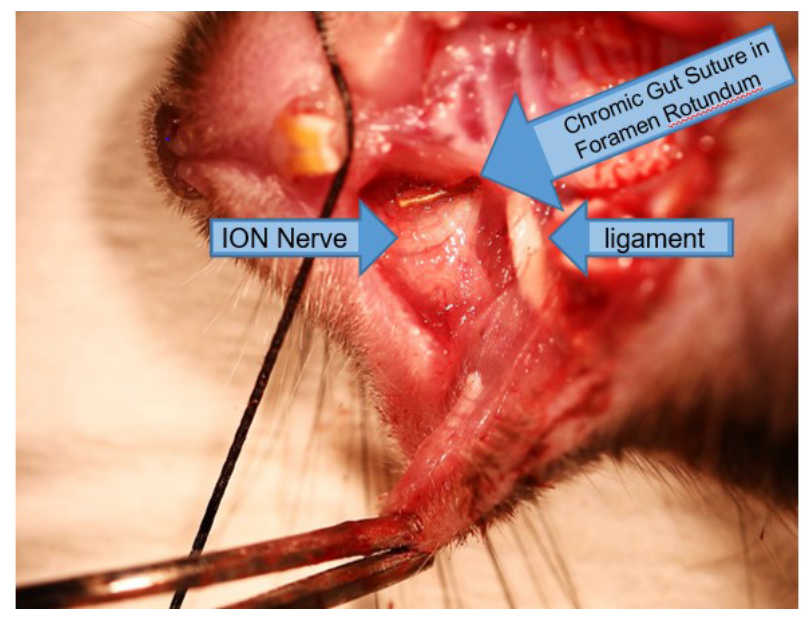

Figure 9. Insertion of chromic gut suture into the foramen rotundum alongside the ION nerve $(15 x)$

12. Using small forceps (recommended: Dumont \#5), grip $3 \mathrm{~mm}$ suture by the end and angle it into the hole you have just cut in the crease of the mouse's cheek (Figure 8). Then slide the suture into the foramen, alongside the ION. The suture should go in with very little resistance; if you feel resistance, it means that you do not have the right angle and are not guiding the suture into the foramen. The nerve should never be touched with the metal surgical instruments.

13. Push the suture into the foramen as far as possible with the forceps (Figures 8 and 9). Then, gently let go, and use the closed tips of your forceps to push the end of the suture further through the foramen. You should just barely be able to see the end of the suture if you have pushed it in correctly.

14. The incision used in this model is small enough that no sutures are required to close the wound. In both shams and the FRICT-ION model, the wound begins to close within the first twenty-four hours (Figure 10). 


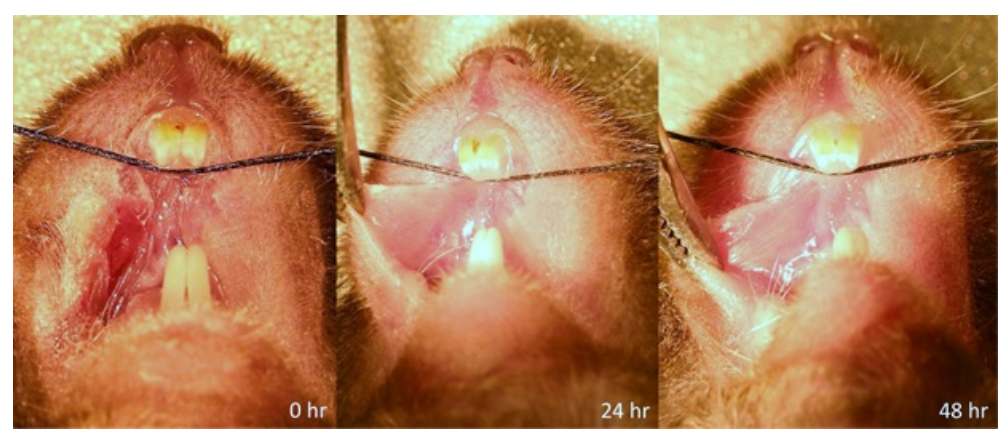

Figure 10. Sham Surgery at 0,24 , and $48 \mathrm{~h}$ post surgery. Wound is mostly closed within $24 \mathrm{~h}$ and is completely closed by $48 \mathrm{~h}$. Only slight vascularity can be seen at $24 \mathrm{~h}$.

15. Place the mouse into the heat recovery station until it awakes from the anesthesia and is mobile before returning to the home cage. Mice should be checked an hour after surgery to ensure all normal motor function has returned. (This is a precaution, as typically mice are fully functional within minutes of removal from anesthesia.) Mice should then be checked daily for the week following surgery, including assessing weights and surface glances into the mouth to ensure no exacerbation of the wound or infection has occurred. It is likely that mice will not gain weight the day following the surgery, but by day two should be back to the typical gradual weight gain.

There should be no noticeable change in behavior; FRICT-ION mice should appear similar to naïve mice to the naked eye. If there is a change in behavior, such as weight loss, lethargy, etc., do not use the mouse for further experimentation. If the weight falls below eighty percent of the mouse's weight prior to surgery, the mouse should be checked by veterinary staff and, if applicable, humanely euthanized.

\section{Data analysis}

\section{Nociceptive and anxiety related behavioral testing and data analysis}

Animals are moved from the housing room and acclimated in the testing room for $30 \mathrm{~min}$ in their home cages prior to testing. Animals are acclimated to gentle restraint (held in gloved hand for whisker pad tests) to assess sensitivity to mechanical stimulation applied to the whiskerpad, i.e., reflexive withdrawal. After 10 training sessions to acclimate the rodent to being held in a gloved hand, sensitivity of the face to mechanical is quantified by the number of withdrawal events from graded thin nylon von Frey filaments with defined bending forces (tensile strength). Stimulation with the lowest fiber $(0.008 \mathrm{~g}, 1.65)$ is not detectable on the back of the human hand; the largest fiber ( $6.0 \mathrm{~g}, 4.74)$ provides the sensation of a blunt paper clip wire end. Animals are free to voluntarily move their head away from the stimulus. A single trial consists of 5 applications of von Frey filaments beginning mid-range $(0.4 \mathrm{~g}, 3.61)$ applied once every 3 to $4 \mathrm{~s}$. If a positive response occurs, the next weaker filament is applied instead, continuing until there is no response; if no positive response is evoked, the next stronger filament is applied and continues until there is a response (Chaplan et al., 1994). The mean occurrence of withdrawal events in each of the trials is expressed as the 
number of responses out of 5, 0 indicates no withdrawal, and 5 indicates the maximum number of withdrawals. Responses to decreased gram force compared to controls indicate increased sensitivity. After induction of trigeminal nerve injury models, statistically significant mechanical hypersensitivity develops reliably within a week and persists indefinitely as tested with calibrated von Frey filaments in the ION's whisker pad receptive field (Figure 11). The mechanical stimulation threshold on both the ipsilateral and contralateral whisker pads is significantly decreased in mice after the surgery compared to surgical sham and control mice. This decrease persists until euthanasia, ten weeks post-surgery.

\section{Von Frey Application on Surgery Side}

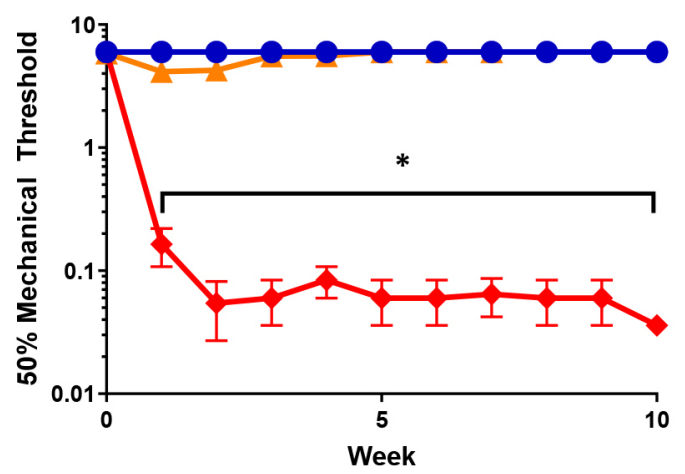

Von Frey Application on Contralateral Side

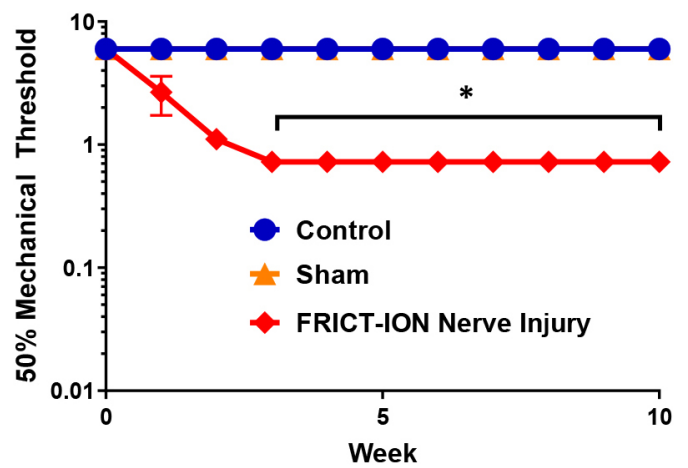

Figure 11. Male mice were tested for mechanical allodynia on the whisker pad using von

Frey filaments. Two-way ANOVA (Dunnett's multiple comparisons test) was performed on these data: $n=5$ per group, ${ }^{*}$ indicates a $P$-value of $<0.05$ [Surgery side: $F(20,198)=9.178$, Contralateral side: $F(22,144)=32.20]$. In post-hoc analyses, Bonferroni adjustment to all $P$ values for week-by-week comparisons of FRICT-ION versus Control yields all nine $P$-values < 0.0009

While management of neuropathic pain is important because in many cases it is severe and unrelenting, study to develop better treatment of the anxiety and depression comorbidities is also of prime importance in overall quality of life (Moulin et al., 2007). Another major advantage of the FRICT-ION model is the ability to examine these behaviors at chronic time points. Testable in the model are anxiety-related measures such as zero maze, light-dark preference, and open field exploratory behaviors. In Figure 12, male mice were placed into a light/dark chamber and monitored for ten minutes. In this test, one chamber was lighted with a bright bulb while the other was kept dark, and mice were allowed free access to either chamber. Mice with FRICT-ION surgery were significantly less likely to spend time in the light chamber, and explored the chamber less in general, which is indicative of anxiety. 


\section{Total Occupancy Time (Light)}

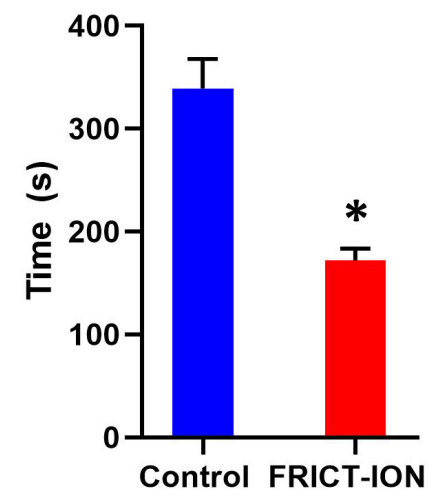

Figure 12. Anxiety behaviors shown were tested in week 8 post model induction $(\mathrm{n}=5$ per group, ${ }^{*}$ indicates a $P$-value of $<0.05$, comparisons run using a $t$-test).

Both cognitive and the sucrose splash depression test (Figure 13) can be utilized to differentiate these higher order consequences of chronic pain in the FRICT-ION model (Yalcin et al., 2011; Kniffin et al., 2015). Mice were given the sucrose splash test, in which $30 \%$ sucrose water is sprayed onto mouse's rump, and mice were monitored for five minutes to determine the number of times they groomed the rump and cage area wetted. Mice with FRICT-ION surgery groomed significantly less often, which is indicative of depression behavior.

\section{Number of Times Groomed}

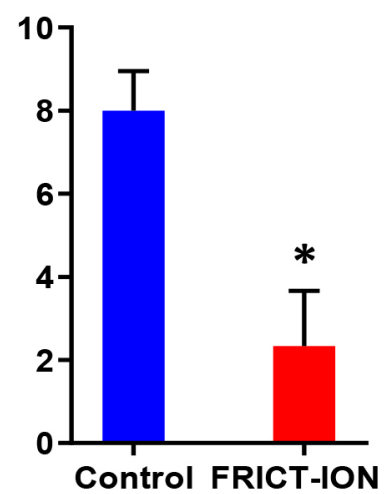

Figure 13. Depression behaviors were tested in week 8 post model induction in male mice ( $n=5$ per group, ${ }^{*}$ indicates a $P$-value of $<0.05$, comparisons run using a $t$-test).

\section{$\underline{\text { Notes }}$}

1. For the sham surgery, the small cut is made in the bucchal margin but no suture inserted. If done correctly, this process should not induce any bleeding inside the mouse's mouth or brain cavity. Remove the mouse from its restraints and place on a paper towel in a clean cage with bedding that has been sitting on a heating pad. The mouse should become conscious less than a minute after you have removed it from anesthesia, and should be up and walking normally 
within five minutes. The mouse should recover from this surgery with ease, and be back to normal behavior by the end of the day.

2. With practice, this procedure should take no more than a few minutes. It is important to perform this procedure quickly to avoid the side effects of long-term exposure to anesthesia.

3. This procedure should be done with no bleeding; however, if bleeding occurs, simply apply pressure to the site with a cotton swab until the bleeding subsides.

4. Mice can be housed together, and should not require any care after surgery except for standard monitoring.

\section{Acknowledgments}

The authors wish to acknowledge the laboratory management skills of Katherine Gott. Departmental funds and NIH grant R21 DE028096 support the Anesthesiology Department Pain Laboratory. The minimally invasive intraoral FRICT-ION approach described here was first used in a recent publication, but we referred to it there as the "TIC" method (Zhang et al., 2019). The FRICT-ION protocol was adapted or modified from our previous trigeminal inflammatory compression (TIC) method where chromic gut suture was aligned along the infraorbital nerve in mice using the more invasive surgical approach behind the eye (Ma et al., 2012 and 2015; Lyons et al., 2015 and 2018). The authors acknowledge the insight provided by former research scientist, Dr. Fei Ma, who introduced the concept of inducing infraorbital nerve compression by suture insertion into a foramen rather than tying the nerve to produce a mouse preclinical model of chronic trigeminal neuropathic pain.

\section{Competing interests}

KNW is an unpaid advisor to USA Elixiria Biotech Inc. and a Research Physiologist at the Albuquerque VA Health Care System. This communication does not necessarily reflect the views of the Department of Veterans Affairs or the U.S. government.

\section{Ethics}

All studies were designed to minimize animal use and performed in accordance with institutional approvals and in accordance with the National Institute of Health Guide for the Care and Use of Laboratory Animals (NIH Publications No. 80-23) revised 1996.

\section{References}

1. Carlson, C. R. (2007). Psychological factors associated with orofacial pains. Dent Clin North Am 51(1): 145-160, vii. 
2. Chaplan, S. R., Bach, F. W., Pogrel, J. W., Chung, J. M. and Yaksh, T. L. (1994). Quantitative assessment of tactile allodynia in the rat paw. J Neurosci Methods 53(1): 55-63.

3. Chen, J., Lee, S., Lui, T., Yeh, Y., Chen, T. and Tzaan, W. (2000). Teflon granuloma after microvascular decompression for trigeminal neuralgia. Surg Neurol 53(3): 281-287.

4. Dandy, W. E. (1934). Concerning the cause of trigeminal neuralgia. Am J Surg 24(2): 447-455.

5. Ding, W., You, Z., Shen, S., Yang, J., Lim, G., Doheny, J. T., Chen, L., Zhu, S. and Mao, J. (2017). An improved rodent model of trigeminal neuropathic pain by unilateral chronic constriction injury of distal infraorbital nerve. J Pain 18(8): 899-907.

6. Dutta, S. and Sengupta, P. (2016). Men and mice: Relating their ages. Life Sci 152: 244-248.

7. Hannaman, M. R., Fitts, D. A., Doss, R. M., Weinstein, D. E. and Bryant, J. L. (2016). The refined biomimetic NeuroDigm GEL model of neuropathic pain in a mature rat. F1000Res 5: 2516.

8. Hardt, S., Fischer, C., Vogel, A., Wilken-Schmitz, A. and Tegeder, I. (2019). Distal infraorbital nerve injury: a model for persistent facial pain in mice. Pain 160(6): 1431-1447.

9. Imamura, Y., Kawamoto, H. and Nakanishi, O. (1997). Characterization of heat-hyperalgesia in an experimental trigeminal neuropathy in rats. Exp Brain Res 116(1): 97-103.

10. Kniffin, T. C., Danaher, R. J., Westlund, K. N., Ma, F., Miller, C. S. and Carlson, C. R. (2015). Persistent neuropathic pain influences persistence behavior in rats. J Oral Facial Pain Headache 29(2): 183-192.

11. Lyons, D. N., Kniffin, T. C., Zhang, L. P., Danaher, R. J., Miller, C. S., Bocanegra, J. L., Carlson, C. R. and Westlund, K. N. (2015). Trigeminal Inflammatory Compression (TIC) injury induces chronic facial pain and susceptibility to anxiety-related behaviors. Neuroscience 295: 126-138.

12. Lyons, D. N., Zhang, L., Pandya, J. D., Danaher, R. J., Ma, F., Miller, C. S., Sullivan, P. G., Sirbu, C. and Westlund, K. N. (2018). Combination drug therapy of pioglitazone and Dcycloserine attenuates chronic orofacial neuropathic pain and anxiety by improving mitochondrial function following trigeminal Nerve Injury. Clin J Pain 34(2): 168-177.

13. Ma, F., Zhang, L., Lyons, D. and Westlund, K. N. (2012). Orofacial neuropathic pain mouse model induced by Trigeminal Inflammatory Compression (TIC) of the infraorbital nerve. $M o l$ Brain 5: 44.

14. Ma, F., Zhang, L., Oz, H. S., Mashni, M. and Westlund, K. N. (2015). Dysregulated TNFalpha promotes cytokine proteome profile increases and bilateral orofacial hypersensitivity. Neuroscience 300: 493-507.

15. Moulin, D. E., Clark, A. J., Gilron, I., Ware, M. A., Watson, C. P., Sessle, B. J., Coderre, T., Morley-Forster, P. K., Stinson, J., Boulanger, A., Peng, P., Finley, G. A., Taenzer, P., Squire, P., Dion, D., Cholkan, A., Gilani, A., Gordon, A., Henry, J., Jovey, R., Lynch, M., Mailis-Gagnon, A., Panju, A., Rollman, G. B., Velly, A. and Canadian Pain, S. (2007). Pharmacological management of chronic neuropathic pain - consensus statement and guidelines from the Canadian Pain Society. Pain Res Manag 12(1): 13-21. 
16. Rodriguez, E., Sakurai, K., Xu, J., Chen, Y., Toda, K., Zhao, S., Han, B. X., Ryu, D., Yin, H., Liedtke, W. and Wang, F. (2017). A craniofacial-specific monosynaptic circuit enables heightened affective pain. Nat Neurosci 20(12): 1734-1743.

17. Vos, B. P., Strassman, A. M. and Maciewicz, R. J. (1994). Behavioral evidence of trigeminal neuropathic pain following chronic constriction injury to the rat's infraorbital nerve. J Neurosci 14(5 Pt 1): 2708-2723.

18. Wang, D. D., Raygor, K. P., Cage, T. A., Ward, M. M., Westcott, S., Barbaro, N. M. and Chang, E. F. (2018). Prospective comparison of long-term pain relief rates after first-time microvascular decompression and stereotactic radiosurgery for trigeminal neuralgia. $J$ Neurosurg 128(1): 6877.

19. Weis, J., Brandner, S., Lammens, M., Sommer, C. and Vallat, J. M. (2012). Processing of nerve biopsies: a practical guide for neuropathologists. Clin Neuropathol 31(1): 7-23.

20. Xia, L., Zhong, J., Zhu, J., Wang, Y. N., Dou, N. N., Liu, M. X., Visocchi, M. and Li, S. T. (2014). Effectiveness and safety of microvascular decompression surgery for treatment of trigeminal neuralgia: a systematic review. J Craniofac Surg 25(4): 1413-1417.

21. Yalcin, I., Barthas, F. and Barrot, M. (2014). Emotional consequences of neuropathic pain: insight from preclinical studies. Neurosci Biobehav Rev 47: 154-164.

22. Yalcin, I., Bohren, Y., Waltisperger, E., Sage-Ciocca, D., Yin, J. C., Freund-Mercier, M. J. and Barrot, M. (2011). A time-dependent history of mood disorders in a murine model of neuropathic pain. Biol Psychiatry 70(10): 946-953.

23. Zhang, M., Hu, M., Montera, M. A. and Westlund, K. N. (2019). Sustained relief of trigeminal neuropathic pain by a blood-brain barrier penetrable PPAR gamma agonist. Mol Pain 15: 1744806919884498. 